\title{
Addressing Implementation Challenges to Digital Care Delivery for Adults With Multiple Chronic Conditions: Stakeholder Feedback in a Randomized Controlled Trial
}

Kelly Williams ${ }^{1 *}$, MPH, PhD; Sarah Markwardt ${ }^{1 *}$, MID; Shannon M Kearney ${ }^{1 *}$, CPH, MPH, DrPH; Jordan F Karp ${ }^{2 *}$, MD; Kevin L Kraemer ${ }^{2 *}$, MD; Margaret J Park ${ }^{3 *}$, MDiv; Paul Freund ${ }^{4 *}$, MEd; Andrew Watson ${ }^{5 *}$, FACS, M. Litt, MD; James Schuster $^{1 *}$, MBA, MD; Ellen Beckjord ${ }^{1 *}, \mathrm{PhD}, \mathrm{MPH}$

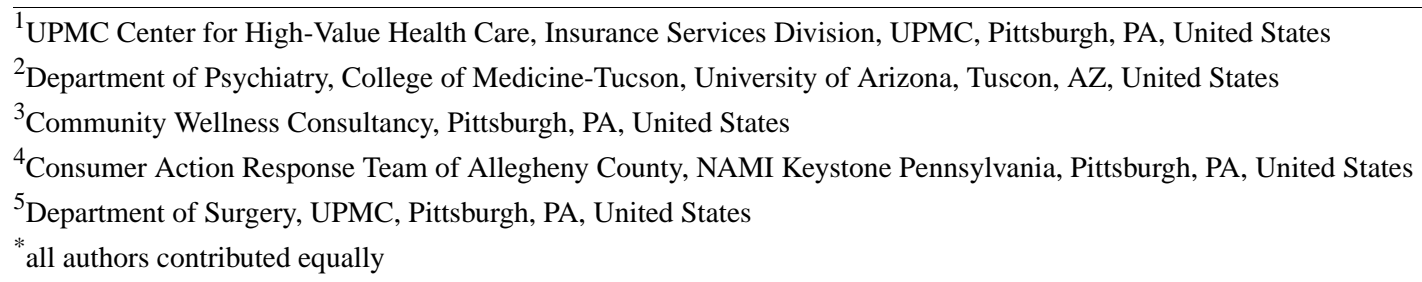

Corresponding Author:

Kelly Williams, MPH, PhD

UPMC Center for High-Value Health Care

Insurance Services Division

UPMC

600 Grant St

Floor 40, Center for High-Value Health Care

Pittsburgh, PA, 15219

United States

Phone: 14124541198

Email:williamsk17@upmc.edu

\section{Abstract}

Background: Digital tools accessed via smartphones can promote chronic condition management, reduce disparities in health care and hospital readmissions, and improve quality of life. However, whether digital care strategies can be implemented successfully on a large scale with traditionally underserved populations remains uncertain.

Objective: As part of a randomized trial comparing care delivery strategies for Medicaid and Medicare-Medicaid beneficiaries with multiple chronic conditions, our stakeholders identified implementation challenges, and we developed stakeholder-driven adaptions to improve a digitally delivered care management strategy (high-tech care).

Methods: We used 4 mechanisms (study support log, Patient Partners Work Group log, case interview log, and implementation meeting minutes) to capture stakeholder feedback about technology-related challenges and solutions from 9 patient partners, 129 participants, and 32 care managers and used these data to develop and implement solutions. To assess the impact, we analyzed high-tech care exit surveys and intervention engagement outcomes (video visits and condition-specific text message check-ins sent at varying intervals) before and after each solution was implemented.

Results: Challenges centered around 2 themes: difficulty using both smartphones and high-tech care components and difficulty using high-tech care components due to connectivity issues. To respond to the first theme's challenges, we devised 3 solutions: tech visits (eg, in-person technology support visits), tech packet (eg, participant-facing technology user guide), and tailored condition-specific text message check-ins. During the first 20 months of implementation, 73 participants received at least one tech visit. We observed a $15 \%$ increase in video call completion for participants with data before and after the tech visit $(\mathrm{n}=25)$ and a $7 \%$ increase in check-in completion for participants with data before and after the tech visit $(n=59)$. Of the 379 participants given a tech packet, 179 completed care during this timeframe and were eligible for an exit survey. Of the survey respondents, $76 \%$ (73/96) found the tech packet helpful and 64\% (62/96) actively used it during care. To support condition-specific text message check-in completion, we allowed for adaption of day and/or time of the text message with 31 participants changing the time they received check-ins and change in standard biometric settings with 13 physicians requesting personalized settings for participants. 
To respond to the second theme's challenges, tech visits or phone calls were made to demonstrate how to use a smartphone to connect or disconnect from the internet, to schedule video calls, or for condition-specific text message check-ins in a location with broadband/internet.

Conclusions: Having structured stakeholder feedback mechanisms is key to identify challenges and solutions to digital care engagement. Creating flexible and scalable solutions to technology-related challenges will increase equity in accessing digital care and support more effective engagement of chronically ill populations in the use of these digital care tools.

Trial Registration: ClinicalTrials.gov NCT03451630; https://clinicaltrials.gov/ct2/show/NCT03451630.

(JMIR Mhealth Uhealth 2021;9(2):e23498) doi: 10.2196/23498

\section{KEYWORDS}

telehealth tools; smartphones; remote patient monitoring; care management; mobile phone

\section{Introduction}

Over the last 10 years, ownership and use of smartphones has more than doubled in the United States, from $35 \%$ to $81 \%$ of the population [1]. One potential benefit of increased access to smartphones is the reduction in health disparities. As smartphone ownership becomes more equitable across socioeconomic categories [1], the use of smartphones provides an opportunity for traditionally underserved or isolated populations to remain connected to health care professionals despite geographic distance or mobility limitations, to quickly receive up-to-date and accurate health education information, and to monitor changes in health conditions using digital health care strategies [2]. In the context of care management teams, remote monitoring platforms provide the opportunity to scale programs, allowing teams of health care professionals and social workers to reach a higher number of individuals living in medically underserved areas [3].

Digital care tools have a growing evidence base, including evidence supporting the effectiveness of such technologies for patients managing chronic conditions. For example, these tools can support individuals with diabetes in lowering hemoglobin $\mathrm{A}_{1 \mathrm{c}}$ levels, improve quality of life and lower number of hospital readmissions for individuals with heart failure [4], improve symptoms and outcomes for individuals with respiratory conditions [5], support better blood pressure control for individuals with hypertension [6], and reduce symptoms of depression [7]. Increased access to and use of digital care strategies has the potential to increase health systems' ability to achieve the quadruple aim: improving population health, enhancing both patient and provider experience, and reducing costs [8].

Despite increased smartphone use and evidence supporting the benefits of digital care strategies such as remote patient monitoring, biosensors, and wearable devices, barriers to care are ever-present when implementing digital tools on a large scale. Notably, there is a lack of research on the challenges that occur when implementing digital care with traditionally underserved populations and those with high-burden, high-cost medical conditions [9]. A pervasive barrier to the success of digital tools is that individuals may lack confidence in their ability to learn how to use these new tools, which may impact their readiness to engage with such tools [10]. According to recent research on mobile devices and health, over half of
Americans are considered to have low digital literacy skills when it comes to using mobile devices [11]. A recent survey of Americans aged above 65 years indicated that although respondents generally had a positive view of technology, they doubted their capacity to learn to use new technology without extra help [12]. In addition, although access to smartphones is becoming more prevalent among all socioeconomic groups, a digital divide still exists in the United States between high- and low-income Americans [13,14], with some research showing that both access and ability may be contributing reasons for why low-income adults may use online health resources less $[15,16]$. Finally, research has historically focused on remote patient monitoring care effectiveness with older Americans [17] and may not address common challenges faced by younger and other diverse populations.

Overcoming these challenges to realize the full potential of digital care to support the management of chronic conditions and reduce health disparities requires an iterative development approach that includes ongoing consumer and community stakeholder input. We are conducting a large-scale, randomized controlled trial comparing the effectiveness of 3 care management strategies (ie, high-touch, high-tech, and usual care) delivered by a commercial insurance organization for adult Medicaid and Medicare-Medicaid beneficiaries living with multiple chronic conditions. To address the unique needs and challenges experienced by this population and to ensure that our digital interventions are patient-centered and pragmatic, we describe early implementation challenges and our stakeholder-driven process adaptions specific to the digitally delivered chronic disease care management strategy (high-tech care).

\section{Methods}

\section{Randomized Controlled Trial Overview}

The 3 study comparators are approaches to care delivered by a chronic disease care management program and incorporate fundamental, evidence-based components of integrated care models including interdisciplinary care management [18,19], individualized care plans [18,19], chronic disease self-management education or self-care support [8,18,20-24], and linkages to medical/behavioral health and social services $[18,25,26]$. High-touch is delivered primarily face-to-face, with telephonic support as needed. High-tech is delivered via a remote care management platform. Both high-touch and 
high-tech participants receive care management for at least 4 months and can continue care for up to 1 year, based on need. Usual care consists of an initial visit and care management for 14 days, which includes connections to condition management support and resources. Care managers receive a weekly worklist denoting individuals who are eligible to be offered participation in the study. To be eligible, individuals must be 21 years or older, have Medicaid or Medicare-Medicaid insurance, have at least 2 chronic conditions, including 1 physical health condition, and have been discharged from a hospital within the past 30 days.

\section{Introducing Participants to the High-tech Care Strategy}

All study participants work, one on one, with a care manager (ie, nurse, social worker, and licensed professional counselor) to create individualized care plans [18,19] centered around chronic disease self-management education, self-care support [8,18,20-24], and to form linkages to medical, behavioral health, and social services $[18,25,26]$. Participants in the high-tech care management strategy have an initial face-to-face appointment with their care manager and are provided with a preconfigured iPhone that allows for care to continue digitally via a remote monitoring platform. We provide iPhones to participants to ensure access to smartphones, and the cost of cellular data is not a barrier to participation. At the initial appointment, the care manager explains 2 key components of the remote monitoring platform that the participant will use on a regular basis: video visits (eg, video conferencing between patients and care managers) and condition-specific text message check-ins. The remote monitoring platform facilitates video conferencing (eg, video visits) between participants and their care manager. Moreover, as our study population has multiple physical and behavioral health conditions, condition-specific check-in questions are sent via text messages to each participant at varying intervals (eg, daily, weekly, or biweekly) based on their condition(s). Check-ins allow care managers to monitor diverse participant needs, symptoms, or condition exacerbations, including specific biometric readings such as pulse, blood glucose level, weight, and blood pressure.

\section{Stakeholder Engagement and Feedback Processes}

At the onset of implementation, the study team was acutely aware of the need for continued stakeholder engagement and feedback to promote effective high-tech care implementation. On the basis of the Patient-Centered Outcome Engagement principles [27], we developed 4 mechanisms (Textbox 1) to capture feedback and input from various stakeholder groups. Patients, care managers, and clinical leadership all provided key insights and observations related to technology challenges that care managers or participants experienced during high-tech care implementation. This stakeholder input was collated from the study support log, the case interview log, and the implementation meeting minute $\log$ (Textbox 1). Feedback was iteratively reviewed by the study and the clinical team. Stakeholder input was organized by topic and content to understand early stage implementation challenges. Topics were then reviewed by the study team and organized into 2 major thematic categories; themes were reviewed with key stakeholders for validation.

Using information from these 3 feedback mechanisms, we developed solutions to the identified technology-related challenges. Solutions were discussed, refined, and implemented with input from the study team, care managers, and clinical leadership. Solutions were also vetted through the Patient Partners Work Group. A work group of patient partners, who have similar characteristics and lived experiences similar to those experienced by our study population, was established through a collaboration with the National Alliance on Mental Illness Southwestern Pennsylvania's Consumer Action Response Team. Patient partner feedback was tracked in the Patient Partners Work Group log (Textbox 1).

Textbox 1. Mechanisms to capture stakeholder feedback on challenges and/or solutions.

Data source and information collected and provided:

- Study support log

- Study team created a study-specific, toll-free, hotline staffed during office hours

- Hotline supports care managers and participants with study-related questions or challenges

- Implementation meeting minutes

- $\quad$ Study team meets with clinical leadership weekly and meets monthly with all care managers

- Meetings provide a time and space for care managers and their clinical leadership to voice implementation challenges and to strategize potential solutions

- Case interview log

- Semistructured interviews were conducted with care managers to identify technology-related challenges, participants experience, and workflow impacts

- $\quad$ Patient Partners Work Group log

- Study team meets regularly with the work group to discuss high-tech care implementation

- The work group provides feedback on materials and solutions supporting high-tech care engagement/implementation 


\section{Understanding Process Modifications: Sources of Information}

In order to assess a change in participants' abilities to overcome technology-related challenges, we analyzed intervention engagement outcomes that may have been impacted by stakeholder-driven implementation solutions. We reviewed the following 3 sources of engagement data pertaining to care activity from April 23, 2018, to December 31, 2019: (1) the participants' ability to complete a video visit as defined by answering the video call from their care manager, (2) the participants' ability to answer condition-specific text message check-ins, as defined by receiving a check-in via text message and submitting all answers to condition-specific questions, and (3) participant responses to exit survey questions sent via the remote monitoring platform. All pre- and postdata presented are based on the first in-person technology support visit completed by the participant.

\section{Results}

\section{Overview}

Stakeholders reported challenges centered around 2 major themes: (1) difficulties using basic functionalities of the smartphone and high-tech care components and (2) difficulties using high-tech care components due to cellular reception and internet connectivity issues. Approximately 500 study hotline phone calls, about technology-specific challenges, were made by 129 participants and 32 care managers to the study team, and the calls were tracked in the study support log. Feedback was also provided during clinical leadership meetings $(n=82)$, monthly care management staff meetings $(n=16$, tracked via implementation meeting minutes), and semistructured interviews with care managers $(n=4$, tracked via case interview log). For each thematic challenge, we present: (1) specific stakeholder feedback that leads to solution development, (2) the description of the stakeholder-driven solutions as they are a direct result of stakeholder feedback, and (3) changes in participant engagement data after solution implementation.

\section{Theme 1 Challenge: Smartphone and High-tech Care Digital Component Use}

With support from care managers and clinical leadership, the study team focused on common functionality challenges experienced by high-tech care participants and devised 3 main solutions: (1) tech visits (technology support visits), (2) a tech packet (participant-facing technology user guide), and (3) tailored condition-specific text message check-in. Table 1 displays stakeholder feedback regarding the participants' experiences when using the smartphone and high-tech care components.

Table 1. Sources of information and solutions: utilizing functionalities of the smartphone and high-tech components.

\begin{tabular}{|c|c|c|}
\hline Data source & Information provided & Informed solution \\
\hline $\begin{array}{l}\text { Case interview log and study } \\
\text { support log }\end{array}$ & $\begin{array}{l}\text { 1. Care managers concerned about time spent teaching participants basic smart- } \\
\text { phone functionalities } \\
\text { 2. Specific technology challenges faced by participants include screen pressure } \\
\text { difficulties, home screen navigation, phone charging, text message access, } \\
\text { including opening condition-specific check-ins, and phone volume manipula- } \\
\text { tion }\end{array}$ & $\begin{array}{l}\text { 1. Tech visits and tech packet } \\
\text { 2. Tech packet }\end{array}$ \\
\hline
\end{tabular}

Implementation meeting minutes

Study support $\log$
1. Clinical leadership interpreted technology-education time concerns as a workflow issue in which care managers had to make up time to ensure participant clinical care needs were met

1. Smartphone factory resets and reconfiguration were time consuming for care managers

2. Smartphone volume manipulation and battery power were 2 participant challenges that resulted in missed high-tech care video visits and condition-specific check-ins

3. Check-in assignments were sometimes automatically scheduled at inconvenient times for participants (ie, work, school or sleeping hours)

4. Biometric check-in settings were automatically standardized for each participant
1. Tech visits and tech packet

1. Tech visits and tech packet

2. Tech packet

Tailored condition-specific check-ins

4. Tailored condition-specific check-ins

\section{Technology Support Visit (Tech Visit) Solution for Theme 1: Description and Changes in Engagement}

Tech visits are structured to allow a study team member to assist participants with time-consuming digital literacy challenges, either at the participant's home or at a community location. Tech visits do not replace initial face-to-face training that care managers provide to high-tech care participants; rather, it is a form of supplemental training to ensure that participants are able to use their smartphone to receive care and to reduce the time care managers spend on high-tech care training during the initial appointment. Participants are selected for tech visits if (1) they have called the study hotline multiple times with issues that could not be completely resolved, (2) a care manager is unable to connect with the member due to technology challenges, and (3) clinical supervisors believe a participant's level of digital literacy requires a substantial amount of care manager's time.

During the first 20 months of implementation, 73 participants received at least one tech visit. Before the tech visit, 23\% (17/73) of the participants completed a video call with their care 
manager. Within 30 days of a tech visit, the average rate for video call completion increased to $51 \%(n=33)$. In total, 21 members, who had never been able to connect with their care manager via video calls, before the tech visit completed a video call after receiving the tech support. Of the 73 participants, 25

Figure 1. Video call data for participants with completed pre- and postdata. CM: care manager.
(34\%) had video call data for both before and after the tech visit. For these participants, the average rate of completed calls increased by $15 \%$ after the completion of a tech visit (Figure $1)$.

\section{Before tech visit}

\section{After tech visit}

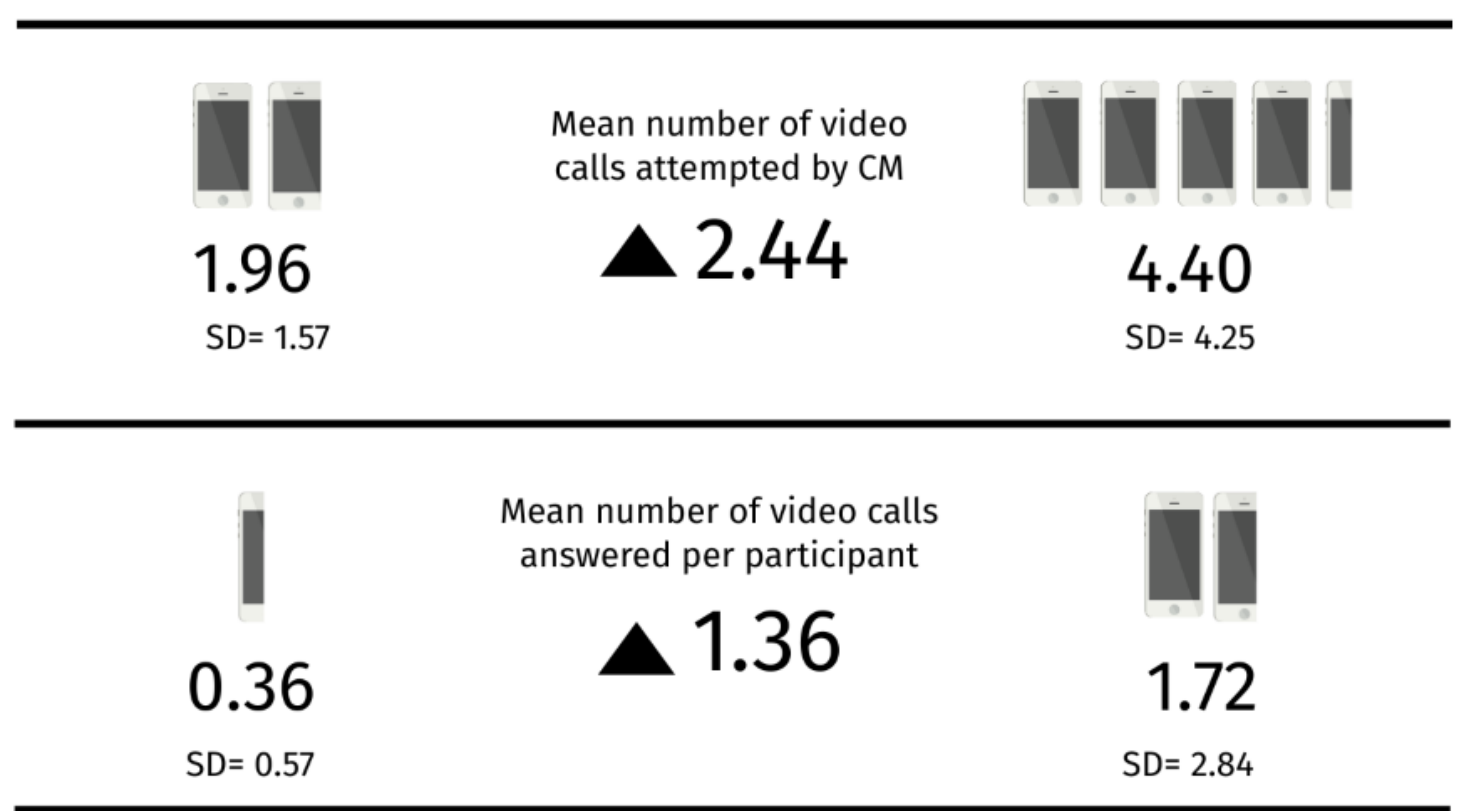

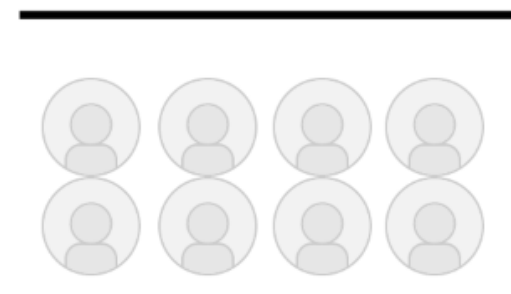

8

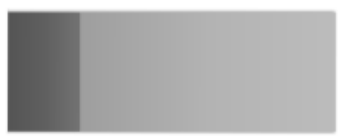

$22 \%$

$\mathrm{SD}=0.37$
Average rate of video calls completed with a participant

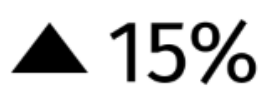

16

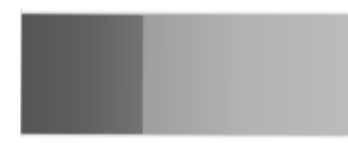

$37 \%$

$\mathrm{SD}=0.39$

Data for participants with whom CM attempted calls both before and after tech visit. $(\mathrm{N}=25)$

Note:

Indicates increase

\section{SD indicates standard deviation}

Specific to condition-specific check-ins sent via text message, most participants completed at least one check-in on their own before their first tech visit $(60 / 73,82 \%)$, with an average rate of $36 \%(n=59)$. Within 30 days of a tech visit, the average rate for engagement with check-ins increased to $43 \%(n=59)$. Two participants completed check-ins after receiving support, who had never completed a check-in prior. Conversely, 15 participants completed check-ins before receiving support but 
never completed a check-in after the visit. Of the 73 participants, $59(81 \%)$ had check-in data both before and after the tech visit.
For these participants, the average rate of completed check-ins increased by $7 \%$ after the completion of a tech visit (Figure 2).

Figure 2. Check-in data for participants with completed pre- and postdata.

\section{Before tech visit}

\section{After tech visit}

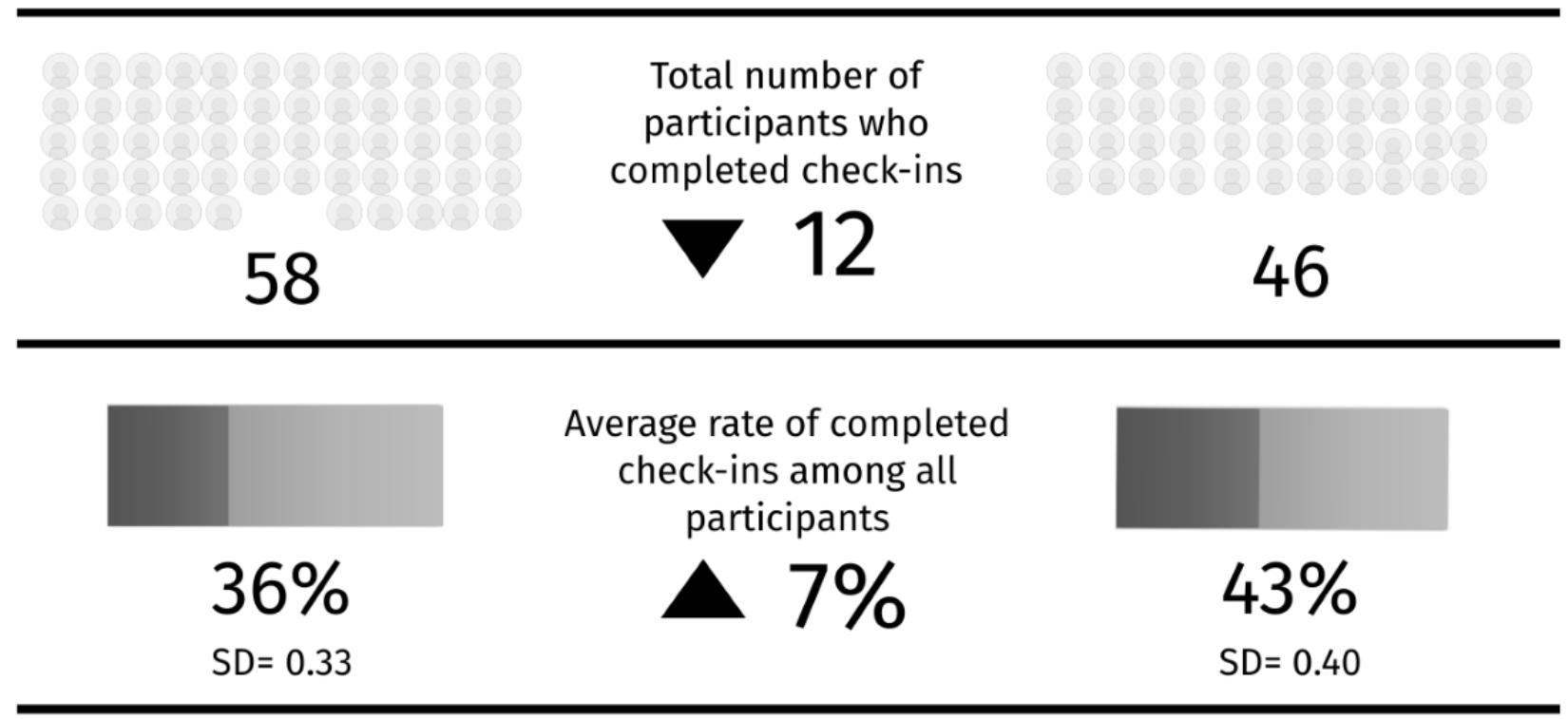

Data for participants who received check-in messages both before and after tech visit. $(\mathrm{N}=59)$

\section{Note:}

Indicates increase

Indicates decrease

\section{SD indicates standard deviation}

\section{Participant-Facing Technology User Guide (Tech Packet) Solution for Theme 1: Description and Changes in Engagement}

The participant-facing technology user guide (tech packet) is an educational resource for participants learning how to use the smartphone and high-tech care digital components. The tech packet outlines key smartphone functions (ie, how to answer a phone call, how to navigate to the home screen, how to open a text message, etc) and high-tech digital care components (ie, how to answer a video call and how to respond to a condition-specific check-in). Care managers give the tech packet to participants during the initial appointment when the participant is randomized into high-tech care; care managers explain the tech packet and have participants practice key functions that they will use throughout their care. Figure 3 shows 2 pages from the tech packet that were developed in response to specific challenges reported in the study support log (Table 1). Feedback from our patient partners, members of the Patient Partners Work Group, was collected over 2-hour-long meetings. Table 2 displays the Patient Partners Work Group feedback on the solution and details on how their feedback was incorporated, and Figure 4 presents a visual example of feedback incorporation. The tech packet is updated regularly based on participant, patient partner, care manager, and clinical leadership feedback. 
Figure 3. Tech packet additions based on study support log.
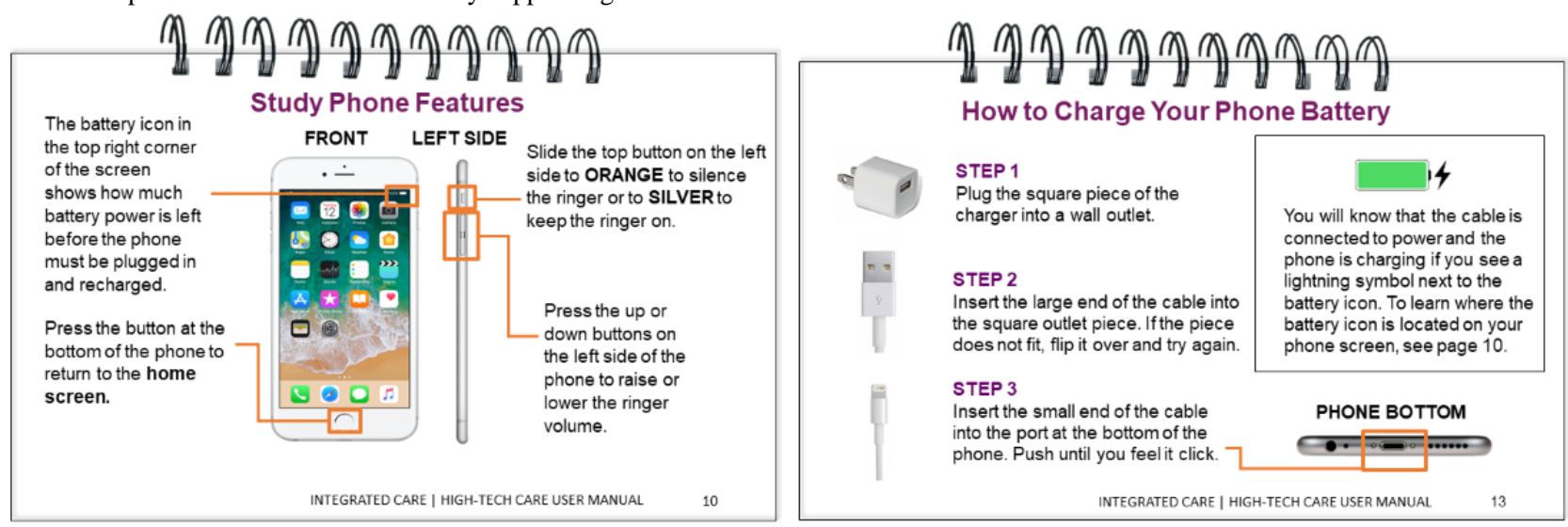

Table 2. Patient Partners Work Group technology user guide feedback.

\begin{tabular}{|c|c|c|}
\hline Work group feedback & Examples of feedback & Feedback incorporated \\
\hline $\begin{array}{l}\text { Use less abbreviations/ jar- } \\
\text { gon as these are difficult to } \\
\text { follow }\end{array}$ & $\begin{array}{l}\text { 1. App has many meanings to different participants } \\
\text { 2. Remote monitoring may have a negative connotation for } \\
\text { participants }\end{array}$ & $\begin{array}{l}\text { 1. Addition of definitions page and spelling out } \\
\text { abbreviations: app to application } \\
\text { 2. Replacement of technical jargon: Check-ins } \\
\text { with your care manager instead of remote } \\
\text { monitoring }\end{array}$ \\
\hline $\begin{array}{l}\text { Provide an easy start point } \\
\text { for each section; assume the } \\
\text { lowest level of digital litera- } \\
\text { cy when creating the instruc- } \\
\text { tions }\end{array}$ & $\begin{array}{l}\text { 1. Each section begins on the smartphone home screen, which } \\
\text { assumes participants can find the home screen }\end{array}$ & $\begin{array}{l}\text { 1. Addition of instructions on how to navigate } \\
\text { to home screen at the beginning and end of } \\
\text { each section }\end{array}$ \\
\hline $\begin{array}{l}\text { Dexterity and pressure diffi- } \\
\text { culties may be a concern }\end{array}$ & 1. Press, click, touch, and open were used interchangeably & $\begin{array}{l}\text { 1. Used touch for screen actions and press for } \\
\text { the home screen button to distinguish amount } \\
\text { of pressure to be applied }\end{array}$ \\
\hline $\begin{array}{l}\text { Highlight important contact } \\
\text { information associated with } \\
\text { care management and study } \\
\text { activities }\end{array}$ & $\begin{array}{l}\text { 1. Participants may not be able to distinguish who is sending a } \\
\text { text message, and some may be concerned about the legitima- } \\
\text { cy of messages }\end{array}$ & $\begin{array}{l}\text { 1. Stated explicitly messages from the remote } \\
\text { monitoring platform come from the same } \\
\text { phone number each time and make the number } \\
\text { visible on all sections } \\
\text { 2. Displayed the study hotline number frequently }\end{array}$ \\
\hline $\begin{array}{l}\text { Participants can be difficult } \\
\text { to reach }\end{array}$ & 1. Participants may not be reached during business hours & $\begin{array}{l}\text { 1. Included a section on how to set up and check } \\
\text { a voicemail box }\end{array}$ \\
\hline
\end{tabular}


Figure 4. Tech packet updates pre- and postpatient Partners Work Group feedback.

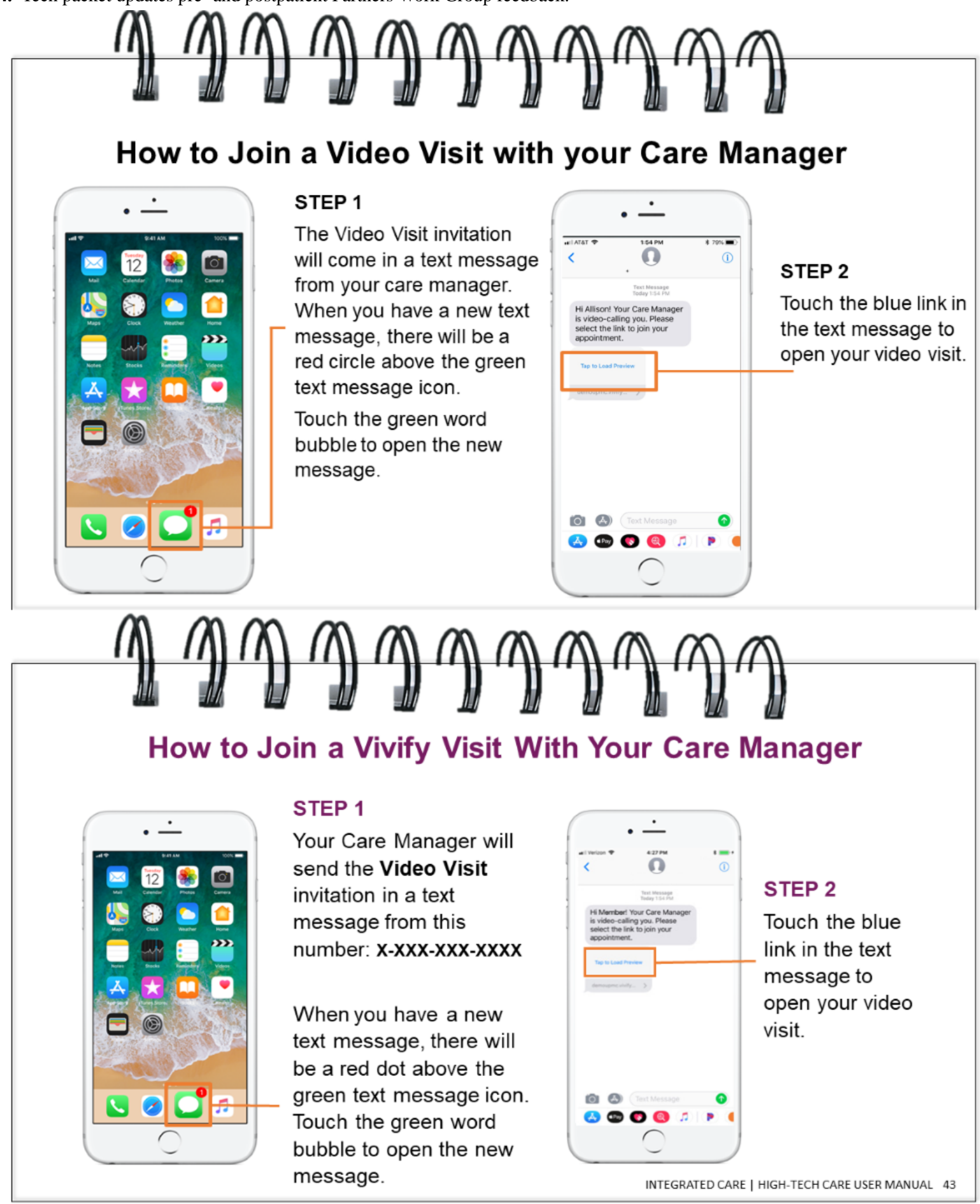

We distributed the tech packets to 379 participants. Of the individuals who received a packet, 179 participants were eligible (eg, completed high-tech care before January 1, 2020) to receive the high-tech care exit survey. Of the 179 participants, 96 responded to the survey; 73 respondents strongly agreed or agreed that the tech packet was useful and 62 actively used the guide at least 1 to 2 times a week during care. See Figure 5 for detailed exit survey results. 
Figure 5. High-tech care exit survey $(96 / 179,53.6 \%)$.

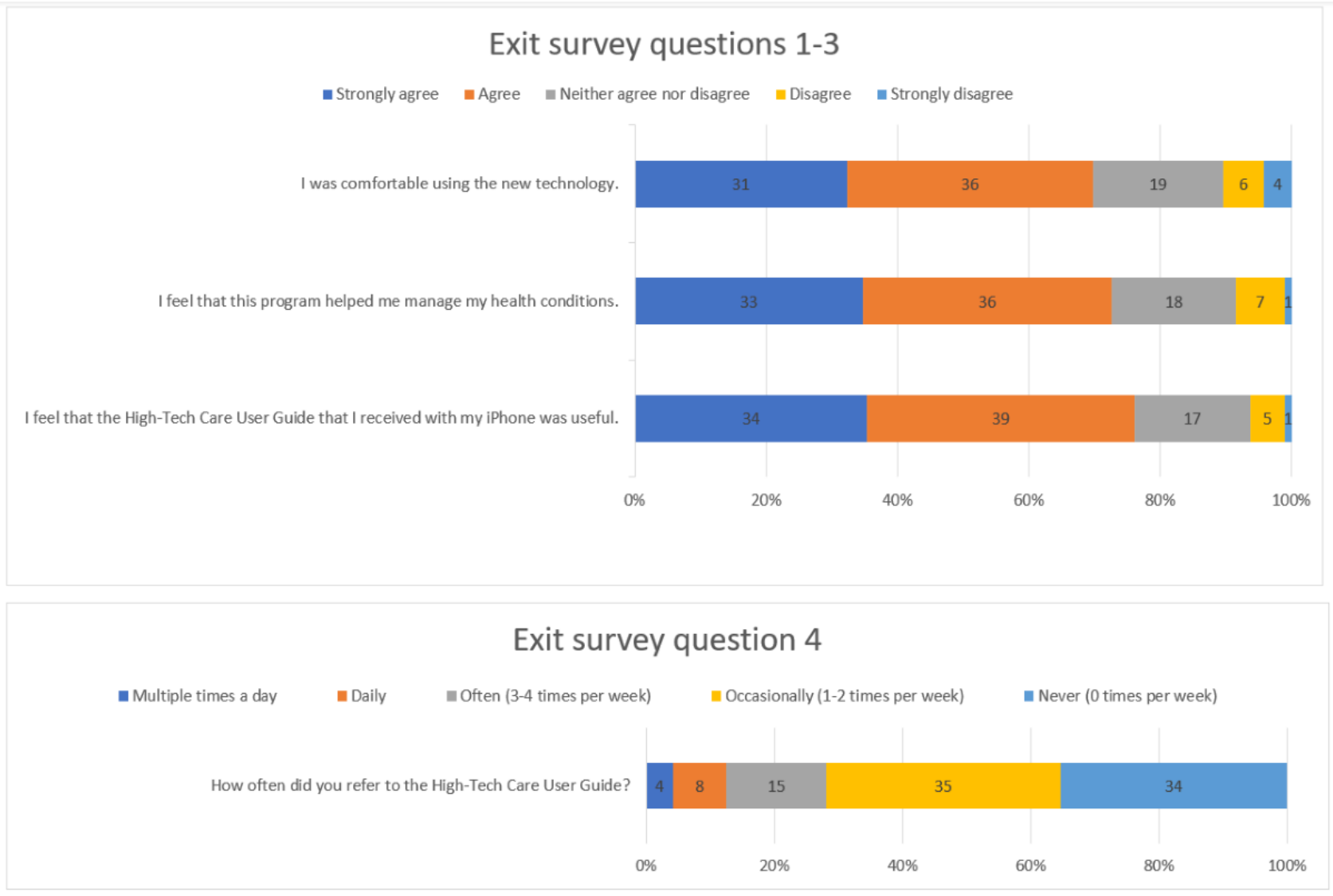

\section{Check-in Tailoring Solution for Theme 1: Description and Impact}

Not all challenges presented to the study team were solved through tech visits and the tech packet. To support participants in completing their condition-specific check-ins, the study team adapted their workflow to allow for a modification of when check-ins are assigned (ie, day of the week and time) based on participant preference. In total, 31 participants requested to change the day/time when check-in text messages were to be received. Second, as all biometric check-in settings (eg, normal boundary parameters for pulse, blood glucose level, weight, and blood pressure) were standardized across participants by default, the study team allowed individual check-in settings to be modified based on the agreement of a participant's primary medical provider. For 13 participants, the primary medical provider requested biometric setting changes to reflect the participant's normal range; this modification allows care managers to better track if biometric readings fall outside the participant's expected range. Textbox 2 describes specific cases in which check-in assignments or biometric settings were modified.

Textbox 2. Condition-specific check-in tailoring examples.

- Check-in assignments modified based on participant preferences

- Participant rescheduled their diabetes check-in for the morning, based on when their medical provider had instructed them to check their glucose

- Participant rescheduled their check-in assignment to the day they are off work

- $\quad$ Biometric settings modified based on provider preferences

- Primary medical provider verified that the participant takes glucose readings before taking insulin and requested setting alerts to be set at 270 or above

- Primary medical provider approved to change a participant's blood pressure settings; allowing notification to only send to the care manager when the participant is out of their expected range $>170 / 100$ or $<90 / 60$

Theme 2 Challenge: Limited Cellular Reception and Internet Connectivity

It was reported, by both care managers and participants themselves, that participants were having difficulties using high-tech care digital components (eg, video visits and condition-specific check-ins) due to limited cellular reception and internet connectivity. Depending on the circumstances, solutions include (1) participant education on how to connect 
a smartphone to the internet, (2) schedule video visits or condition-specific check-ins when the participant is in a location with cellular service or internet, and (3) participant education on how to disconnect from the internet. Table 3 displays the sources of information that led the study team to become aware of the challenge participants experienced with connectivity.

Table 3. Sources of information and solutions: understanding limited cellular reception and intervention connectivity.

\begin{tabular}{|c|c|c|}
\hline Data source & Information provided & Informed solution \\
\hline $\begin{array}{l}\text { Study support log and imple- } \\
\text { mentation meeting minutes }\end{array}$ & $\begin{array}{l}\text { - Server error messages reported by participants preventing check-in messages } \\
\text { from opening }\end{array}$ & $\begin{array}{l}\text { Education on connecting a smart- } \\
\text { phone to the internet }\end{array}$ \\
\hline $\begin{array}{l}\text { Study support log and imple- } \\
\text { mentation meeting minutes }\end{array}$ & $\begin{array}{l}\text { - No internet at the home preventing digital tool use } \\
\text { - No cellular reception at home prevented digital tool use }\end{array}$ & $\begin{array}{l}\text { Scheduling video visits/check-ins } \\
\text { when participant has access to } \\
\text { cellular service or internet }\end{array}$ \\
\hline $\begin{array}{l}\text { Study support log and imple- } \\
\text { mentation meeting minutes }\end{array}$ & $\begin{array}{l}\text { Poor internet service at home chosen as default connection method prevented } \\
\text { digital tool use } \\
\text { Poor internet service in community chosen as default connection method pre- } \\
\text { vented digital tool use }\end{array}$ & $\begin{array}{l}\text { Education on disconnecting from } \\
\text { the internet }\end{array}$ \\
\hline
\end{tabular}

\section{Solutions for Theme 2: Description and Changes in Engagement}

Care managers communicate with each participant regarding the best way to provide care, given limited cellular reception and internet connectivity. All 3 connectivity solutions are addressed via a tech visit or phone call. Providing education on connecting a smartphone to the internet is often a solution for participants receiving a server error message when attempting to open condition-specific check-ins. Participants were advised to connect to the home internet in case of a bandwidth issue. Scheduling video visits and condition-specific check-ins at specific times affords participants the knowledge that they will have access to cellular service or internet and has been another viable solution. For example, one participant rescheduled their condition-specific check-ins to days of the week when a routine visit was set with family members who have internet access or to business hours as their employer offers internet access. Finally, providing education to participants on how to disconnect from the internet can support video visits or check-in completion. For example, participants are instructed to disconnect weak internet connections, such as home connections or public connections, when they have strong cellular service.

\section{Discussion}

\section{Principal Findings}

This paper highlights the impact of stakeholder-driven solutions on early implementation challenges specific to a digital care strategy. To support participants in engaging with their smartphone and high-tech care digital components (theme 1 challenge), 3 main solutions were implemented (eg, in-person technology support visits, participant-facing technology user guides, and tailored condition-specific check-ins). For participants who received an in-person technology support visit, we saw an overall increase in engagement with video calls and condition-specific check-ins. For participants who received the participant-facing technology user guide and completed both high-tech care and the exit survey, we found that most used the tech packet while receiving care and or believed it was useful. Finally, condition-specific check-ins were tailored for participants to support engagement and meet their primary medical provider's care goals. To support participants experiencing difficulties engaging in high-tech care due to limited cellular reception or internet connectivity (theme 2 challenges), 3 solutions were devised and implemented as needed to support engagement in the program (eg, education on both connecting and disconnecting a smartphone to the internet, scheduling high-tech care video visits or condition-specific check-ins at times when the participant is in a physical location that allows connectivity to occur).

Our findings suggest that concurrent stakeholder feedback has the potential to increase implementation success; therefore, it is pivotal to provide stakeholders with multiple and continuous avenues for communicating challenges to the study team. Furthermore, the results stress the importance of working collaboratively with stakeholders early in the implementation of digital interventions to design scalable solutions such as educational materials (tech packet) and activities (tech visits and telephonic support) and refine condition-specific check-ins that suit the specific needs of the patient and their primary medical provider. Moreover, although measuring the success of solutions created during implementation is not always preplanned, early results indicate positive changes in participant technology engagement after tech visits are implemented. Our positive trends in engagement highlight the need for earlier identification of patients who require tech visits to promote early engagement, reduce demoralization, and potentially achieve earlier clinical benefits. Understanding the nuanced challenges of delivering interventions and engaging patients-as well as how to create effective solutions-will advance the efficiency and reach of digital care.

\section{Comparison With Prior Work}

Current digital care literature focuses on either how tool engagement impacts desired health outcome(s) [6] or defining tool use metrics [28,29]. Processes and solutions to overcome tool utilization barriers are underdeveloped topics in the field that has implications for replication and scaling. One systematic review of digital mental health interventions targeting college students found that of the 89 studies, 45 reported outcomes focused on usability and acceptability (many with low rates of 
response) and only 2 studies reported on feasibility [30]. It is critical to expand knowledge centered on how to design and adapt implementation processes in order for digital care teams to be equipped with the right knowledge and resources to best overcome challenges to digital care provision for chronically ill and low-income populations. Providers delivering care digitally must understand and be able to adequately address patient-specific barriers to using digital care tools before patients can engage in the evidence-based tool functions and work toward improved health outcomes.

Our work is an important addition to the discussion on digital care provision as we provide a systematic framework for how digital care providers can work with stakeholder groups to identify and address care delivery implementation challenges. Although most research on remote patient monitoring focuses on single-diagnosis care for older Americans, our intervention targets adults aged 21 years and older who are managing multiple chronic physical and or behavioral health conditions [6]. Our findings expand knowledge beyond the traditional populations included in digital health research.

Finally, as participants in this research are exclusively eligible for Medicaid, our work promises to reduce health disparities by improving access to digitally delivered evidence-based care management for low-income patients. Supporting consumer adoption of digital health tools is one way to both support patients in their management of chronic conditions and the ethical imperative of reducing health disparities. However, realizing the full potential of digital tools to positively impact health disparities requires continued work on understanding the ways to best support traditionally underserved populations to use digital tools (and how to design those tools and their implementation protocols to meet diverse consumer needs).

\section{Limitations}

Although this study contributes novel stakeholder-driven solutions to stakeholder-reported implementation challenges that affect participants' engagement in a digital care intervention, there are several limitations. Data collected via the high-tech care exit survey may be limited due to a nonresponse bias, as participants who completed the survey had to be able to access the check-in and be willing to complete the survey after completing all care goals. However, our current response rate of $54 \%$ indicates that this bias may be less salient [31]. In addition, we were not able to control for additional factors such as in-home caregiver support, improvement in health conditions, or time participating in care that may have also impacted a participant's ability to overcome the specific challenges that our solutions were designed to target. However, as our solutions were codesigned with key stakeholders and our ability to review pre- and postsolution data, it is reasonable to assume that our solutions influenced positive trends in high-tech care engagement. We also acknowledge that our provision of a smartphone to all study participants may be perceived as a potential barrier to scalability. However, it is important to understand engagement-related challenges for individuals with varying levels of experience with such technology and provide the same phone to all study participants allows us to understand how heterogeneity in technology comfort/experience manifests over the course of the intervention. For future efforts, care managers can and do support individuals with the procurement of a government-issued smartphone that has similar functionality to the phone provided for this study; thus, our findings related to technology engagement can likely be generalized beyond the scope of this study.

\section{Conclusions}

To better understand digital care barriers specific to a patient population or care program, it is critical to develop and employ methods for obtaining feedback from key stakeholders before and during implementation. Key stakeholders may include care providers and implementation teams, digital tool creators, and community organizations that represent the population of interest and patients. Our stakeholder-informed solutions include in-person tech visits, a tech packet detailing how to access and use key technology components, along with the tailoring of digital care components to meet both patient and provider needs. Using high-tech care exit survey responses and remote monitoring engagement data, we measured the impact of our solutions and continued to improve high-tech care delivery. As solutions to challenges develop, detailed tracking of their implementation may positively impact patient engagement with digital tools and ultimately show increased participation in care resulting in improved health outcomes and reductions in health outcome disparities.

\section{Acknowledgments}

The authors thank all care managers, clinical leadership, Patient Partners Work Group, Stakeholder Advisory Board, and our study participants for their collaboration and input into each stage of the study thus far and for their continued commitment to this research. The authors also thank the study team for their dedication and preserving adaptability. ClinicalTrials.gov identifier: NCT03451630. This study was partially funded by the Patient-Centered Outcomes Research Institute (PCORI). All statements in this report, including its findings and conclusions, are solely those of the authors and do not necessarily represent the views of PCORI, its Board of Governors or Methodology Committee. The participation of JS in the research project occurred before JS's appointment to the Board of Governors of the PCORI (R).

\section{Conflicts of Interest}

JK has received medication supplies for investigator-initiated trials from Pfizer and Indivior. He received compensation for an educational webinar from Otsuka (condition-specific, not product-focused) within the past 12 months. He receives ongoing compensation for editorial work from the American Association for Geriatric Psychiatry and Physicians Postgraduate Press. 


\section{References}

1. Mobile fact sheet. Pew Research Center. 2019. URL: https://www.pewresearch.org/internet/fact-sheet/mobile/ [accessed 2020-12-28]

2. Vivify H. Managing chronic disease. Vivify Health Published. 2018. URL: https://www.vivifyhealth.com/download/ chronic-disease/ [accessed 2020-12-28]

3. Shea S, Weinstock RS, Starren J, Teresi J, Palmas W, Field L, et al. A randomized trial comparing telemedicine case management with usual care in older, ethnically diverse, medically underserved patients with diabetes mellitus. J Am Med Inform Assoc 2006;13(1):40-51 [FREE Full text] [doi: 10.1197/jamia.M1917] [Medline: 16221935]

4. Bashi N, Karunanithi M, Fatehi F, Ding H, Walters D. Remote monitoring of patients with heart failure: an overview of systematic reviews. J Med Internet Res 2017 Jan 20;19(1):e18 [FREE Full text] [doi: 10.2196/jmir.6571] [Medline: $\underline{28108430}$ ]

5. Au DH, Macaulay DS, Jarvis JL, Desai US, Birnbaum HG. Impact of a telehealth and care management program for patients with chronic obstructive pulmonary disease. Ann Am Thorac Soc 2015 Mar;12(3):323-331. [doi: 10.1513/AnnalsATS.201501-042OC] [Medline: 25642649]

6. Flodgren G, Rachas A, Farmer AJ, Inzitari M, Shepperd S. Interactive telemedicine: effects on professional practice and health care outcomes. Cochrane Database Syst Rev 2015 Sep 07(9):CD002098 [FREE Full text] [doi: 10.1002/14651858.CD002098.pub2] [Medline: 26343551]

7. Firth J, Torous J, Nicholas J, Carney R, Pratap A, Rosenbaum S, et al. The efficacy of smartphone-based mental health interventions for depressive symptoms: a meta-analysis of randomized controlled trials. World Psychiatry 2017 Oct;16(3):287-298 [FREE Full text] [doi: 10.1002/wps.20472] [Medline: 28941113]

8. Bodenheimer T, Wagner EH, Grumbach K. Improving primary care for patients with chronic illness. JAMA 2002 Oct 09;288(14):1775-1779. [doi: 10.1001/jama.288.14.1775] [Medline: 12365965 ]

9. Safavi K, Mathews SC, Bates DW, Dorsey ER, Cohen AB. Top-funded digital health companies and their impact on high-burden, high-cost conditions. Health Aff (Millwood) 2019 Jan;38(1):115-123. [doi: 10.1377/hlthaff.2018.05081] [Medline: 30615535]

10. Horrigan JB. The meaning of digital readiness. Pew Research Center. 2016. URL: https://www.pewresearch.org/internet/ 2016/09/20/the-meaning-of-digital-readiness/ [accessed 2020-12-28]

11. Sim I. Mobile devices and health. N Engl J Med 2019 Sep 05;381(10):956-968. [doi: 10.1056/nejmra1806949]

12. Anderson M, Perrin A. Barriers to adoption and attitudes towards technology. Pew Research Center. 2017. URL: https:/ /www.pewresearch.org/internet/2017/05/17/barriers-to-adoption-and-attitudes-towards-technology/ [accessed 2020-12-28]

13. Anderson M, Kumar M. Digital divide persists even as lower-income Americans make gains in tech adoption. Pew Research Center. 2019. URL: https://www.pewresearch.org/fact-tank/2019/05/07/ digital-divide-persists-even-as-lower-income-americans-make-gains-in-tech-adoption/ [accessed 2020-12-28]

14. Bailey SC, O'Conor R, Bojarski EA, Mullen R, Patzer RE, Vicencio D, et al. Literacy disparities in patient access and health-related use of Internet and mobile technologies. Health Expectations 2015 Dec;18(6):3079-3087 [FREE Full text] [doi: 10.1111/hex.12294] [Medline: 25363660]

15. Seo H, Erba J, Altschwager D, Geana M. Evidence-based digital literacy class for older, low-income African-American adults. J Appl Commun Res 2019 Mar 11;47(2):130-152. [doi: 10.1080/00909882.2019.1587176]

16. Jensen JD, King AJ, Davis LA, Guntzviller LM. Utilization of internet technology by low-income adults: the role of health literacy, health numeracy, and computer assistance. J Aging Health 2010 Sep;22(6):804-826. [doi:

10.1177/0898264310366161] [Medline: 20495159]

17. Vegesna A, Tran M, Angelaccio M, Arcona S. Remote patient monitoring via non-invasive digital technologies: a systematic review. Telemed J E Health 2017 Jan;23(1):3-17 [FREE Full text] [doi: 10.1089/tmj.2016.0051] [Medline: 27116181]

18. Bishop M. Facilitators and Barriers to Providing Patient-Centered Chronic Disease Care to Patient Populations at Risk for Health and Health Care Disparities in Safety Net Settings. Essential Hospitals Institute, America's Essential Hospitals. 2013. URL: https://silo.tips/download/essential-hospitals-institute-america-s-essential-hospitals [accessed 2021-01-13]

19. Nolte E, McKee M. Caring for people with chronic conditions - a health systems perspective. In: Gesundheitswesen. Maidenhead, Berkshire: Open University Press; Aug 19, 2009:a.

20. Bodenheimer T, Wagner EH, Grumbach K. Improving primary care for patients with chronic illness: the chronic care model, Part 2. J Am Med Assoc 2002 Oct 16;288(15):1909-1914. [doi: 10.1001/jama.288.15.1909] [Medline: 12377092]

21. Bayliss EA, Steiner JF, Fernald DH, Crane LA, Main DS. Descriptions of barriers to self-care by persons with comorbid chronic diseases. Ann Fam Med 2003;1(1):15-21 [FREE Full text] [doi: 10.1370/afm.4] [Medline: 15043175]

22. Baumann LC, Dang TTN. Helping patients with chronic conditions overcome barriers to self-care. Nurse Pract 2012 Mar 13;37(3):32-38. [doi: 10.1097/01.NPR.0000411104.12617.64] [Medline: 22289883]

23. Wagner EH, Austin BT, Von Korff M. Organizing care for patients with chronic illness. Milbank Q 1996;74(4):511-544. [Medline: $\underline{8941260]}$

24. Clark NM, Becker MH, Janz NK, Lorig K, Rakowski W, Anderson L. Self-Management of Chronic Disease by Older Adults. J Aging Health 2016 Jun 30;3(1):3-27. [doi: 10.1177/089826439100300101] 
25. Crossing the Quality Chasm: a New Health System for the 21st Century. Washington (DC): National Academies Press; 2001.

26. Wagner E. Chronic disease management: what will it take to improve care for chronic illness? Eff Clin Pract 1998;1(1):2-4. [Medline: 10345255]

27. Sheridan S, Schrandt S, Forsythe L, Hilliard TS, Paez KA, Advisory Panel on Patient Engagement (2013 inaugural panel). The PCORI engagement rubric: promising practices for partnering in research. Ann Fam Med 2017 Mar;15(2):165-170 [FREE Full text] [doi: 10.1370/afm.2042] [Medline: 28289118]

28. Krebs P, Sherman S, Wilson H, El-Shahawy O, Abroms LL, Zhao X, et al. Text2Connect: a health system approach to engage tobacco users in quitline cessation services via text messaging. Transl Behav Med 2020 Feb 03;10(1):292-301. [doi: 10.1093/tbm/ibz033] [Medline: 32011721]

29. Cole-Lewis H, Ezeanochie N, Turgiss J. Understanding health behavior technology engagement: pathway to measuring digital behavior change interventions. JMIR Form Res 2019 Oct 10;3(4):e14052 [FREE Full text] [doi: 10.2196/14052] [Medline: 31603427]

30. Lattie EG, Adkins EC, Winquist N, Stiles-Shields C, Wafford QE, Graham AK. Digital mental health interventions for depression, anxiety, and enhancement of psychological well-being among college students: systematic review. J Med Internet Res 2019 Jul 22;21(7):e12869 [FREE Full text] [doi: 10.2196/12869] [Medline: 31333198]

31. Spooner SH. Survey response rates and overall patient satisfaction scores: what do they mean? J Nurs Care Qual 2003;18(3):162-174. [doi: 10.1097/00001786-200307000-00002] [Medline: 12856900]

\section{Abbreviations \\ PCORI: Patient-Centered Outcomes Research Institute}

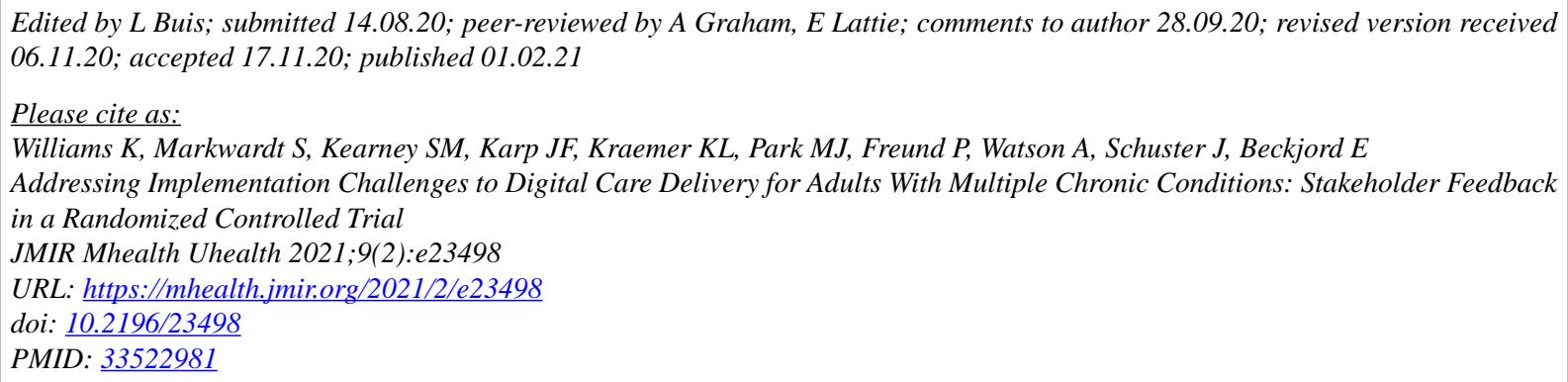

CKelly Williams, Sarah Markwardt, Shannon M Kearney, Jordan F Karp, Kevin L Kraemer, Margaret J Park, Paul Freund, Andrew Watson, James Schuster, Ellen Beckjord. Originally published in JMIR mHealth and uHealth (http://mhealth.jmir.org), 01.02.2021. This is an open-access article distributed under the terms of the Creative Commons Attribution License (https://creativecommons.org/licenses/by/4.0/), which permits unrestricted use, distribution, and reproduction in any medium, provided the original work, first published in JMIR mHealth and uHealth, is properly cited. The complete bibliographic information, a link to the original publication on http://mhealth.jmir.org/, as well as this copyright and license information must be included. 